\title{
An Analytical Air Pollution Model with Time Dependent Eddy Diffusivity
}

\author{
Tiziano Tirabassi $^{{ }^{*},}$ Marco Túllio Vilhena ${ }^{2}$, Daniela Buske ${ }^{3}$, Gervásio Annes Degrazia ${ }^{4}$ \\ ${ }^{1}$ Institute of Atmospheric Sciences and Climate (ISAC), National Research Council (CNR), Bologna, Italy; ${ }^{2}$ Graduate Program in \\ Mechanical Engineering, Federal University of Rio Grande do Sul (UFRGS), Porto Alegre, Brazil; ${ }^{3}$ Department of Mathematics and \\ Statistics (IFM/DME), Federal University of Pelotas (UFPel), Pelotas, Brazil; ${ }^{4}$ Department of Physics, Federal University of Santa \\ Maria (UFSM), Santa Maria, Brazil. \\ Email: "t.tirabassi@isac.cnr.it
}

Received April 30 $0^{\text {th }}, 2013$; revised June $2^{\text {nd }}, 2013$; accepted July $5^{\text {th }}, 2013$

Copyright (C) 2013 Tiziano Tirabassi et al. This is an open access article distributed under the Creative Commons Attribution License, which permits unrestricted use, distribution, and reproduction in any medium, provided the original work is properly cited.

\begin{abstract}
Air pollution transport and dispersion in the atmospheric boundary layer are modeled by the advection-diffusion equation, that is, essentially, a statement of conservation of the suspended material in an incompressible flow. Many models simulating air pollution dispersion are based upon the solution (numerical or analytical) of the advection-diffusion equation assuming turbulence parameterization for realistic physical scenarios. We present the general time dependent three-dimensional solution of the advection-diffusion equation considering a vertically inhomogeneous atmospheric boundary layer for arbitrary vertical profiles of wind and eddy-diffusion coefficients. Numerical results and comparison with experimental data are shown.
\end{abstract}

Keywords: Analytical Solution; Advection-Diffusion Equation; Air Pollution Modeling; Integral Transform; Decomposition Method

\section{Introduction}

The processes governing the transport and diffusion of pollutants present large variability and distinct forms, the phenomenon is of such complexity that it would be impossible to describe it without the use of mathematical models. Such models therefore constitute an indispensable technical instrument of air quality management.

The theoretical approach to the problem essentially assumes different forms. In the $\mathrm{K}$ approach, diffusion is considered, at a fixed point in space, proportional to the local gradient of the concentration of the diffused material. Consequently, it is fundamentally Eulerian since it considers the motion of fluid within a spatially fixed system of reference. They are based on the numerical resolution, on a fixed spatial-temporal grid, of the equation of the mass conservation of the pollutant chemical species, the so-said advection-diffusion equation (ADE).

However, in the last years, much progress has been made in getting an analytical solution of steady state ADE [1]. Recently, the literature presented analytical general solutions of the ADE by the GILTT approach

${ }^{*}$ Corresponding author.
(Generalized Integral Laplace Transform Technique) whose main feature relies on the analytical solution of transformed GITT (Generalized Integral Transform Technique) solutions by the Laplace Transform technique $[2,3]$. This methodology has been largely applied in the topic of simulations of pollutant dispersion in the Atmospheric Boundary Layer (ABL) and is a general steady state solution for any profiles of wind and eddy diffusivity. A new three-dimensional analytical solution is presented in this work for the prediction of pollutant dispersion in the ABL incorporating both the spatial and temporal dependence of the eddy diffusivity.

To accomplish our objective, we solve the temporal dependent three-dimensional advection-diffusion equation combining the Decomposition and GILTT approaches. So far, applying the idea of Decomposition method [4,5], we reduce the ADE with temporal dependence of the eddy diffusivity into a set of recursive ADE's with eddy diffusivity just depending on the spatial variable $z$, which is then directly solved by the GILTT method. We introduced an atmospheric boundary Layer parameterisation with a time dependent vertical eddy diffusivity coefficient and evaluated the performance 
of the proposed model against an experimental data set.

\section{The Analytical Solution}

In the sequel we briefly discuss the solution derivation of the time-dependent, three-dimensional ADE. For such let us consider the problem:

$$
\begin{aligned}
& \frac{\partial \bar{c}}{\partial t}+\bar{u} \frac{\partial \bar{c}}{\partial x}+\bar{v} \frac{\partial \bar{c}}{\partial y}+\bar{w} \frac{\partial \bar{c}}{\partial z} \\
& =\frac{\partial}{\partial x}\left(K_{x} \frac{\partial \bar{c}}{\partial x}\right)+\frac{\partial}{\partial y}\left(K_{y} \frac{\partial \bar{c}}{\partial y}\right)+\frac{\partial}{\partial z}\left(K_{z} \frac{\partial \bar{c}}{\partial z}\right)
\end{aligned}
$$

For $t>0,0<x<L_{x}, 0<y<L_{y}$ and $0<z<h$, subjected to the following boundary and initial conditions:

$$
\begin{aligned}
& K_{z} \frac{\partial \bar{c}}{\partial z}=0 \text { at } z=0, h \\
& K_{y} \frac{\partial \bar{c}}{\partial y}=0 \text { at } y=0, L_{y} \\
& K_{x} \frac{\partial \bar{c}}{\partial x}=0 \text { at } x=0, L_{x} \\
& \bar{c}(x, y, z, 0)=0 \text { at } t=0
\end{aligned}
$$

In the case of horizontal diffusion ( $x, y$ plane), we solve the equation for $x$ and $y$ positive then, being the horizontal dispersion symmetric compared to the $x$ and $y$ axes, the same results are associated with $x$ and $y$ negative coordinates. Here it is assumed that the source term is written as a source condition, quoted as:

$$
\overline{u c}(0, y, z, t)=Q \delta\left(y-y_{0}\right) \delta\left(z-H_{s}\right)
$$

We must notice that $\bar{c}$ denotes the mean concentration of a passive contaminant $\left(\mathrm{g} / \mathrm{m}^{3}\right), \bar{u}, \bar{v}$ and $\bar{w}$ are the cartesian components of the mean wind speed $(\mathrm{m} / \mathrm{s})$ in the directions $x, y$ and $z$, and $K_{x}, K_{y}$ and $K_{z}$ are the eddy diffusivities $\left(\mathrm{m}^{2} / \mathrm{s}\right) . Q$ is the emission rate $(\mathrm{g} / \mathrm{s})$, $\mathrm{h}$ the height of the atmospheric boundary layer $(\mathrm{m}), H_{s}$ the height of the source (m), $L_{x}$ and $L_{y}$ are the limits in the $x$ and $y$-axis and far away from the source $(\mathrm{m})$ and $\delta$ represents the Dirac delta function

In order to solve the problem (1) and also considering the well-known solution of the two-dimensional problem with advection in the $x$-direction by the GILTT method [6,7], we initially apply the general integral transform technique in the $y$ variable. For such, we expand the pollutant concentration as:

$$
\bar{c}(x, y, z, t)=\sum_{m=0}^{\infty} \bar{c}_{m}(x, z, t) Y_{m}(y),
$$

where $Y_{m}(y)$ is a set of orthogonal eigenfunctions, given by $Y_{m}(y)=\cos \left(\lambda_{m} y\right)$ with $\lambda_{m}=m \pi / L_{y} \quad(m=0,1,2, \cdots)$ the respective set of eigenvalues.

To determine the unknown coefficient $\bar{c}_{m}(x, z, t)$ for $m=0 \cdots M$ we begin recasting Equation (1) applying the chain rule for the diffusion terms. It turns out that:

$$
\begin{aligned}
& \sum_{m=0}^{\infty}\left(-\frac{\partial \bar{c}_{m}(x, z, t)}{\partial t} Y_{m}(y)-\bar{u} \frac{\partial \bar{c}_{m}(x, z, t)}{\partial x} Y_{m}(y)\right. \\
& -\overline{v c}_{m}(x, z, t) Y_{m}^{\prime}(y)-\bar{w} \frac{\partial \bar{c}_{m}(x, z, t)}{\partial z} Y_{m}(y) \\
& +K_{x} \frac{\partial^{2} \bar{c}_{m}(x, z, t)}{\partial x^{2}} Y_{n}(y)+K_{x}^{\prime} \frac{\partial \bar{c}_{m}(x, z, t)}{\partial x} Y_{n}(y) \\
& +K_{y} \bar{c}_{m}(x, z, t) Y_{m}^{\prime \prime}(y)+K_{y}^{\prime} \bar{c}_{m}(x, z, t) Y_{m}^{\prime}(y) \\
& \left.+K_{z} \frac{\partial^{2} \bar{c}_{m}(x, z, t)}{\partial z^{2}} Y_{n}(y)+K_{z}^{\prime} \frac{\partial \bar{c}_{m}(x, z, t)}{\partial z} Y_{n}(y)\right)=0
\end{aligned}
$$

Now applying the operator $\int_{0}^{L_{y}} Y_{n}(y)(.) \mathrm{d} y$ to Equation (3) and using the definitions:

$$
\begin{aligned}
& \int_{0}^{L_{y}} Y_{m}(y) Y_{n}(y) \mathrm{d} y=\alpha_{n n}, \int_{0}^{L_{y}} Y_{m}^{\prime}(y) Y_{n}(y) \mathrm{d} y=\beta_{n n} \\
& \int_{0}^{L_{y}} K_{y} Y_{m}(y) Y_{n}(y) \mathrm{d} y=\gamma_{m n}, \int_{0}^{L_{y}} K_{y}^{\prime} Y_{m}^{\prime}(y) Y_{n}(y) \mathrm{d} y=\eta_{m n}
\end{aligned}
$$

the Equation (3) is rewrite as:

$$
\begin{aligned}
& \sum_{m=0}^{\infty}\left(\left[-\frac{\partial \bar{c}_{m}(x, z, t)}{\partial t}+K_{x} \frac{\partial^{2} \bar{c}_{m}(x, z, t)}{\partial x^{2}}\right.\right. \\
& +K_{x}^{\prime} \frac{\partial \bar{c}_{m}(x, z, t)}{\partial x}+K_{z} \frac{\partial \bar{c}_{m}(x, z, t)}{\partial z} \\
& +K_{z} \frac{\partial \bar{c}_{m}(x, z, t)}{\partial z}-\bar{u} \frac{\partial \bar{c}_{m}(x, z, t)}{\partial x} \\
& \left.-\bar{w} \frac{\partial \bar{c}_{m}(x, z, t)}{\partial z}\right] \alpha_{n n}-\bar{v} \beta_{n n} \bar{c}_{m}(x, z, t) \\
& \left.-\lambda_{m}^{2} \gamma_{m n} \bar{c}_{m}(x, z, t)+\eta_{m n} \bar{c}_{m}(x, z, t)\right)=0
\end{aligned}
$$

Making the assumption that the reference system is orientated to the prevailing wind $(\bar{u} \neq 0, \bar{v}=\bar{w}=0)$, and further considering that the advection is dominant in the $x$-direction, the diffusion component $K_{x}$ has been neglected. In addition, it is also considered that $K_{y}$ has only dependence on the $z$-direction [8,9]. These assumptions clearly yield to the ensuing set of $M+1$ two-dimensional diffusion equations:

$$
\begin{aligned}
& \frac{\partial \bar{c}_{m}(x, z, t)}{\partial t}+\bar{u} \frac{\partial \bar{c}_{m}(x, z, t)}{\partial x}-\frac{\partial}{\partial z}\left(K_{z} \frac{\partial \bar{c}_{m}(x, z, t)}{\partial z}\right) \\
& +\lambda_{m}^{2} K_{y} \bar{c}_{m}(x, z, t)=0
\end{aligned}
$$

Now, we are in position to solve Equation (5) following 
the idea of the Decomposition method [4,5]. In fact to construct the solution, firstly it is considered that the time-dependent eddy diffusivity is written like:

$$
K_{z}(z, t)=k_{z}(z, t)+\bar{K}_{z}(z)
$$

where $\bar{K}_{z}(z)$ is the time averaged eddy diffusivity. By this assumption the Equation (5) reads like:

$$
\begin{aligned}
& \frac{\partial \bar{c}_{m}(x, z, t)}{\partial t}+\bar{u} \frac{\partial \bar{c}_{m}(x, z, t)}{\partial x} \\
& -\frac{\partial}{\partial z}\left(\bar{K}_{z}(z) \frac{\partial \bar{c}_{m}(x, z, t)}{\partial z}\right)+\lambda_{m}^{2} K_{y} \bar{c}_{m}(x, z, t) \\
& =\frac{\partial}{\partial z}\left(k_{z}(z, t) \frac{\partial \bar{c}_{m}(x, z, t)}{\partial z}\right)
\end{aligned}
$$

According the idea of the Decomposition method, we consider that the solution of Equation (7) has the form:

$$
\bar{c}_{m}(x, z, t)=\sum_{j=0}^{J} \bar{c}_{m, j}(x, z, t)
$$

Now replacing Equation (8) in Equation (7), from the resulting equation we are able to construct the recursive set of advective-diffusive equations:

$$
\left\{\begin{array}{l}
\frac{\partial \bar{c}_{m, 0}(x, z, t)}{\partial t}+\bar{u} \frac{\partial \bar{c}_{m, 0}(x, z, t)}{\partial x}-\frac{\partial}{\partial z}\left(\bar{K}_{z}(z) \frac{\partial \bar{c}_{m, 0}(x, z, t)}{\partial z}\right) \\
+\lambda_{m}^{2} K_{y} \bar{c}_{m, 0}(x, z, t)=0 \\
\frac{\partial \bar{c}_{m, 1}(x, z, t)}{\partial t}+\bar{u} \frac{\partial \bar{c}_{m, 1}(x, z, t)}{\partial x}-\frac{\partial}{\partial z}\left(\bar{K}_{z}(z) \frac{\partial \bar{c}_{m, 1}(x, z, t)}{\partial z}\right) \\
+\lambda_{m}^{2} K_{y} \bar{c}_{m, 1}(x, z, t)=S_{1}(x, z, t) \\
\vdots \\
\frac{\partial \bar{c}_{m, J}(x, z, t)}{\partial t}+\bar{u} \frac{\partial \bar{c}_{m, J}(x, z, t)}{\partial x}-\frac{\partial}{\partial z}\left(\bar{K}_{z}(z) \frac{\partial \bar{c}_{m, J}(x, z, t)}{\partial z}\right) \\
+\lambda_{m}^{2} K_{y} \bar{c}_{m, J}(x, z, t)=S_{J}(x, z, t)
\end{array}\right.
$$

where we have the following notation for the term $S_{J}$ :

$$
\begin{aligned}
S_{J}(x, z, t)=\frac{\partial}{\partial z}\left(k_{z}(z, t) \frac{\partial \bar{c}_{m, J-1}(x, z, t)}{\partial z}\right) & \\
& \text { for } l=1: J
\end{aligned}
$$

We must recall that this procedure is not unique. We justify our choice in order because this procedure allows us to take advantage from the fact that the resulting recursive system problem can be straightly analytically solved by the GILTT approach $[6,7,10]$. Further we have to notice that the time dependence of the eddy diffusivity in the proposed solution is governed by the source term. It is relevant underline that the first equation of recursive system problems satisfies the boundary conditions (Equations (1a-e)) meanwhile the remaining equations satisfy the homogeneous boundary condition. Once the set of problems (9) is solved by the GILTT method, the solution of Equation (1) is well determined. It is important to remark that we may control the accuracy of the results by a proper choice of the number of terms in the series solution summation.

\section{Model Evaluation against Experimental Data}

The performance of the proposed model is evaluated against the experimental Copenhagen [11,12] data set. In the Copenhagen experiment the tracer $\mathrm{SF}_{6}$ was released without buoyancy from a tower at a height of $115 \mathrm{~m}$, and collected at the ground-level positions at a maximum of three crosswind arcs of tracer sampling units. The sampling units were positioned, at the ground level, $2-6 \mathrm{~km}$ from the point of release. The site was mainly residential with a roughness length of $0.6 \mathrm{~m}$. The meteorological conditions during the dispersion experiments ranged from moderately unstable to convective. We used the values of the maximum concentration on every crosswind arc normalized with the tracer release rate from [11]. Generally, the distributed data set contains hourly mean values of concentrations and meteorological data. However, in this model the validation is performed to show the time dependence of eddy diffusivity using data with a greater time resolution, kindly made available by Gryning and described in [13]. In particular, we used 10 minutes averaged values for meteorological data in Tables 1-3 while Table 4 reported hourly mean values of

Table 1. Friction velocity $\left(u^{*}(\mathrm{~m} / \mathrm{s})\right)$ for different time steps. Each interval corresponds to $10 \mathrm{~min}$.

\begin{tabular}{ccccccccc}
\hline $\mathrm{t} /$ Run & 1 & 2 & 3 & 4 & 5 & 7 & 8 & 9 \\
\hline 1 & 0.36 & 0.68 & 0.46 & 0.56 & 0.58 & 0.48 & 0.65 & 0.72 \\
2 & 0.37 & 0.67 & 0.45 & 0.51 & 0.52 & 0.48 & 0.79 & 0.73 \\
3 & 0.40 & 0.81 & 0.47 & 0.37 & 0.51 & 0.57 & 0.67 & 0.60 \\
4 & 0.43 & 0.68 & 0.39 & 0.44 & 0.58 & 0.62 & 0.67 & 0.59 \\
5 & 0.35 & 0.75 & 0.39 & 0.48 & 0.59 & 0.53 & 0.68 & 0.65 \\
6 & 0.34 & 0.74 & 0.40 & 0.48 & 0.52 & 0.65 & 0.65 & 0.71 \\
7 & 0.42 & 0.76 & 0.40 & 0.39 & 0.52 & 0.63 & 0.68 & 0.73 \\
8 & 0.43 & 0.82 & 0.41 & 0.40 & 0.45 & 0.65 & 0.67 & 0.73 \\
9 & 0.40 & 0.76 & 0.31 & 0.39 & 0.44 & 0.66 & 0.73 & 0.73 \\
10 & 0.37 & 0.73 & 0.34 & 0.39 & 0.44 & 0.62 & 0.73 & 0.66 \\
11 & 0.35 & 0.69 & 0.39 & 0.39 & 0.44 & 0.52 & 0.75 & 0.67 \\
12 & 0.36 & 0.66 & 0.40 & 0.39 & 0.43 & 0.62 & 0.69 & 0.74 \\
\hline
\end{tabular}


Table 2. Convective velocity $\left(w^{*}(\mathrm{~m} / \mathrm{s})\right)$ for different time steps. Each interval corresponds to $10 \mathrm{~min}$.

\begin{tabular}{ccccccccc}
\hline $\mathrm{t} /$ Run & 1 & 2 & 3 & 4 & 5 & 7 & 8 & 9 \\
\hline 1 & 2.07 & 2.04 & 1.21 & 1.32 & 0.93 & 1.93 & 1.99 & 1.35 \\
2 & 2.22 & 1.85 & 1.10 & 1.45 & 1.10 & 1.86 & 2.27 & 1.63 \\
3 & 1.56 & 2.02 & 1.40 & 1.29 & 0.90 & 2.37 & 2.35 & 1.77 \\
4 & 2.11 & 2.11 & 1.18 & 1.37 & 0.82 & 2.15 & 2.32 & 1.43 \\
5 & 1.81 & 2.15 & 1.09 & 1.15 & 1.05 & 1.57 & 2.42 & 1.31 \\
6 & 1.67 & 1.89 & 1.37 & 1.10 & 1.02 & 2.67 & 2.07 & 1.47 \\
7 & 1.98 & 2.39 & 1.29 & 0.89 & 1.02 & 2.37 & 2.49 & 1.51 \\
8 & 2.18 & 2.26 & 1.48 & 0.85 & 0.89 & 2.62 & 2.35 & 1.63 \\
9 & 1.56 & 2.25 & 0.92 & 0.77 & 0.76 & 2.87 & 2.24 & 1.74 \\
10 & 2.29 & 1.69 & 1.38 & 0.77 & 0.76 & 2.15 & 2.31 & 1.59 \\
11 & 1.88 & 2.28 & 1.18 & 0.77 & 0.76 & 1.45 & 2.54 & 1.82 \\
12 & 2.00 & 1.54 & 1.37 & 0.77 & 0.60 & 2.08 & 2.57 & 2.03 \\
\hline
\end{tabular}

Table 3. Monin-Obukhov length (m) for the different runs and time steps. Each time step corresponds to $10 \mathrm{~min}$.

\begin{tabular}{ccccccccc}
\hline $\mathrm{t} /$ Run & 1 & 2 & 3 & 4 & 5 & 7 & 8 & 9 \\
\hline 1 & -26 & -178 & -152 & -75 & -492 & -71 & -71 & -793 \\
2 & -23 & -227 & -194 & -42 & -215 & -80 & -85 & -471 \\
3 & -83 & -311 & -106 & -23 & -368 & -64 & -47 & -202 \\
4 & -42 & -160 & -101 & -32 & -735 & -111 & -49 & -366 \\
5 & -36 & -203 & -129 & -71 & -366 & -177 & -45 & -633 \\
6 & -42 & -286 & -70 & -80 & -273 & -67 & -63 & -588 \\
7 & -47 & -155 & -83 & -83 & -273 & -87 & -41 & -593 \\
8 & -38 & -228 & -60 & -101 & -262 & -71 & -47 & -471 \\
9 & -83 & -184 & -106 & -129 & -395 & -56 & -70 & -389 \\
10 & -21 & -389 & -42 & -129 & -395 & -111 & -64 & -375 \\
11 & -32 & -133 & -101 & -129 & -395 & -215 & -52 & -262 \\
12 & -29 & -375 & -70 & -129 & -759 & -123 & -39 & -252 \\
\hline
\end{tabular}

Table 4. Boundary layer height for the different runs.

\begin{tabular}{ccccccccc}
\hline Run & 1 & 2 & 3 & 4 & 5 & 7 & 8 & 9 \\
\hline $\mathrm{h}(\mathrm{m})$ & 1980 & 1920 & 1120 & 390 & 820 & 1850 & 810 & 2090 \\
\hline
\end{tabular}

boundary layer height.

\section{Boundary Layer Parameterization}

The reliability of the analytical solution of the advectiondiffusion equation depends on the choice of the atmospheric boundary Layer parameterization. In terms of the convective scaling parameters the vertical eddy diffusivity can be formulated as [14]:

$$
\frac{K_{z}}{w_{*} h}=0.22\left(\frac{z}{h}\right)^{\frac{1}{3}}\left(1-\frac{z}{h}\right)^{\frac{1}{3}}\left[1-\mathrm{e}^{\left(-\frac{4 z}{h}\right)}-0.0003 \mathrm{e}^{\left(\frac{8 z}{h}\right)}\right]
$$

The above formulation of the vertical eddy diffusivity varies in time through the time variation of the vertical convective velocity $w^{*}$ and the height of the ABL h. In Figure 1 we show the graphic displaying the vertical eddy diffusivity $K_{z}$ as a sectional function of time for the Copenhagen data set.

For the lateral diffusion we used [14]:

$$
K_{y}=\frac{\sqrt{\pi} \sigma_{v}}{16\left(f_{m}\right)_{v} q_{v}}
$$

with $\sigma_{v}^{2}=\frac{0.98 c_{v}}{\left(f_{m}\right)_{v}^{2 / 3}}\left(\frac{\psi_{\varepsilon}}{q_{v}}\right)^{2 / 3}\left(\frac{z}{h}\right)^{2 / 3} w_{*}^{2} ; \quad q_{v}=4.16 \frac{z}{h} ;\left(f_{m}\right)_{v}=$ 0.16 and $\psi_{\varepsilon}^{1 / 3}=\left[\left(1-\frac{z}{h}\right)^{2}\left(-\frac{z}{L}\right)^{-2 / 3}+0.75\right]^{1 / 2}$. More, $k$ is the von Karman constant $(k=0.4), L$ is the MoninObukhov length, $\sigma_{v}$ is the Eulerian standard deviation of the longitudinal turbulent velocity, $q_{v}$ is the stability function, $\psi_{\varepsilon}$ is the non-dimensional molecular dissipation rate function, $\left(f_{m}\right)_{v}$ is the peak wavelength of the turbulent velocity spectra.

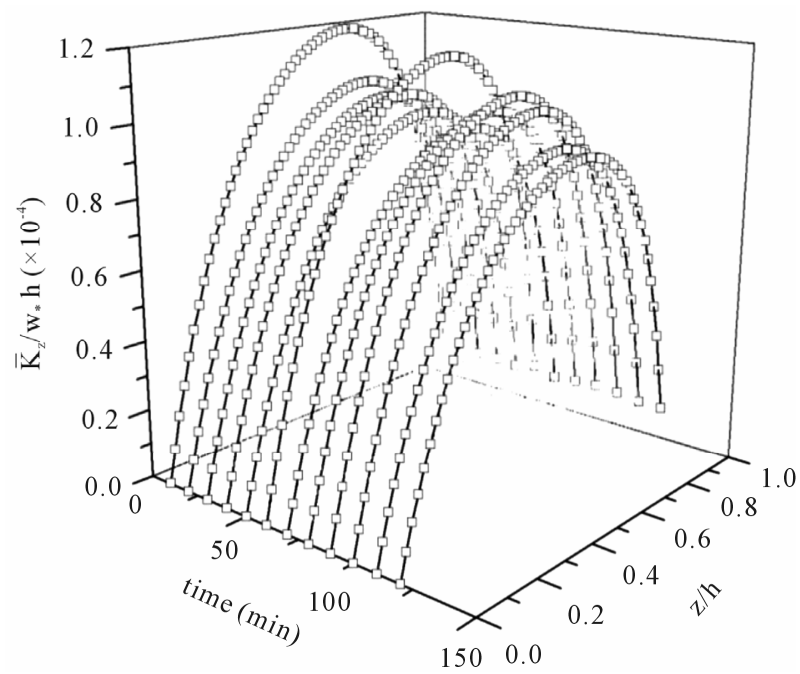

Figure 1. Vertical eddy diffusivity profile $\left(K_{z}\right)$ in function of time for the Copenhagen experimental runs. 
The wind speed profile can be described by a power law expressed as follows [15]:

$$
\frac{\bar{u}_{z}}{\bar{u}_{1}}=\left(\frac{z}{z_{1}}\right)^{n}
$$

where $\bar{u}_{z}$ and $\bar{u}_{1}$ are the mean wind speeds horizontal to heights $z$ and $z_{1}$ and $n$ is an exponent that is related to the intensity of turbulence [16]. For the unstable conditions of the Copenhagen experiment we used $n=0.1$.

\section{Results}

In order to show the performance of the present model and to evaluate the performance of the proposed $\mathrm{ABL}$ parameterization we have applied the model using the Copenhagen experimental data set [11].

The validation has to be considered preliminary one. We have checked the model with data referring to continuous emission in variable meteorology (with time resolution of 10 minutes) and in receptors points far from the source $(2-6 \mathrm{~km})$.

In this work we adopted the value of $J=6$ for the number of recursive problems solved. Indeed, in the sequel we report the results encountered in Table 5 reporting the numerical convergence of the results, considering successively one to six terms in the series solutions. We can observe that the desired accuracy, for the problem solved, is attained with six terms in the series solution, for all distances considered. Once the number of terms in the series solution is known, next in Table 6, we present

Table 5. Numerical convergence of the time-dependent 3DGILTT model for different runs and source distances.

\begin{tabular}{|c|c|c|c|}
\hline \multirow[t]{2}{*}{ Run } & \multirow{2}{*}{$\begin{array}{c}\begin{array}{c}\text { Adomian } \\
\text { recursion } \\
\text { depth }\end{array} \\
0\end{array}$} & \multicolumn{2}{|c|}{$\bar{c}(x, y, z, t) \quad\left(10^{-7} \mathrm{~s} \cdot \mathrm{m}^{-3}\right)$} \\
\hline & & 8.50 & 2.90 \\
\hline \multirow{6}{*}{1} & 1 & 10.19 & 3.26 \\
\hline & 2 & 9.84 & 3.23 \\
\hline & 3 & 9.79 & 3.23 \\
\hline & 4 & 9.79 & 3.23 \\
\hline & 5 & 9.79 & 3.23 \\
\hline & 6 & 9.79 & 3.23 \\
\hline \multirow{7}{*}{2} & 0 & 5.08 & 1.88 \\
\hline & 1 & 5.80 & 2.05 \\
\hline & 2 & 5.65 & 2.03 \\
\hline & 3 & 5.65 & 2.03 \\
\hline & 4 & 5.65 & 2.03 \\
\hline & 5 & 5.65 & 2.03 \\
\hline & 6 & 5.65 & 2.03 \\
\hline
\end{tabular}

\section{Continued}

\begin{tabular}{|c|c|c|c|c|}
\hline & 0 & 12.41 & 4.26 & 2.18 \\
\hline & 1 & 16.83 & 5.18 & 2.55 \\
\hline & 2 & 16.01 & 5.08 & 2.54 \\
\hline \multirow[t]{7}{*}{3} & 3 & 15.72 & 5.07 & 2.54 \\
\hline & 4 & 15.63 & 5.07 & 2.54 \\
\hline & 5 & 15.60 & 5.07 & 2.54 \\
\hline & 6 & 15.61 & 5.07 & 2.54 \\
\hline & 0 & 6.71 & & \\
\hline & 1 & 11.36 & & \\
\hline & 2 & 11.34 & & \\
\hline \multirow[t]{7}{*}{4} & 3 & 11.34 & & \\
\hline & 4 & 11.34 & & \\
\hline & 5 & 11.34 & & \\
\hline & 6 & 11.34 & & \\
\hline & 0 & 11.69 & 4.46 & 2.40 \\
\hline & 1 & 17.52 & 5.73 & 2.92 \\
\hline & 2 & 17.19 & 5.53 & 2.87 \\
\hline \multirow[t]{7}{*}{5} & 3 & 17.15 & 5.48 & 2.87 \\
\hline & 4 & 17.12 & 5.46 & 2.87 \\
\hline & 5 & 16.88 & 5.47 & 2.87 \\
\hline & 6 & 16.17 & 5.47 & 2.87 \\
\hline & 0 & 5.68 & 1.95 & 1.25 \\
\hline & 1 & 6.73 & 2.15 & 1.36 \\
\hline & 2 & 6.53 & 2.12 & 1.35 \\
\hline \multirow[t]{7}{*}{7} & 3 & 6.51 & 2.12 & 1.35 \\
\hline & 4 & 6.51 & 2.12 & 1.35 \\
\hline & 5 & 6.51 & 2.12 & 1.35 \\
\hline & 6 & 6.51 & 2.12 & 1.35 \\
\hline & 0 & 6.74 & 2.55 & 1.44 \\
\hline & 1 & 8.69 & 3.09 & 1.72 \\
\hline & 2 & 8.41 & 3.08 & 1.72 \\
\hline \multirow[t]{7}{*}{8} & 3 & 8.38 & 3.08 & 1.72 \\
\hline & 4 & 8.37 & 3.08 & 1.72 \\
\hline & 5 & 8.37 & 3.08 & 1.72 \\
\hline & 6 & 8.37 & 3.08 & 1.72 \\
\hline & 0 & 5.51 & 2.11 & 1.15 \\
\hline & 1 & 6.24 & 2.29 & 1.23 \\
\hline & 2 & 6.10 & 2.26 & 1.22 \\
\hline \multirow[t]{4}{*}{9} & 3 & 6.09 & 2.26 & 1.22 \\
\hline & 4 & 6.09 & 2.26 & 1.22 \\
\hline & 5 & 6.09 & 2.26 & 1.22 \\
\hline & 6 & 6.09 & 2.26 & 1.22 \\
\hline
\end{tabular}


Table 6. Observed and predicted concentrations data for different runs (Copenhagen experiment) at various source distances. The concentration is divided by the emission rate Q.

\begin{tabular}{|c|c|c|c|}
\hline Run & Distance $(\mathrm{m})$ & $\begin{array}{c}\text { Observed } \\
\left(10^{-7} \mathrm{~s} \cdot \mathrm{m}^{-3}\right)\end{array}$ & $\begin{array}{l}\text { Predictions } \\
\left(10^{-7} \mathrm{~s} \cdot \mathrm{m}^{-3}\right)\end{array}$ \\
\hline 1 & 1900 & 10.5 & 9.79 \\
\hline 1 & 3700 & 2.14 & 3.23 \\
\hline 2 & 2100 & 9.85 & 5.65 \\
\hline 2 & 4200 & 2.83 & 2.03 \\
\hline 3 & 1900 & 16.33 & 15.61 \\
\hline 3 & 3700 & 7.95 & 5.07 \\
\hline 3 & 5400 & 3.76 & 2.54 \\
\hline 4 & 4000 & 15.71 & 11.34 \\
\hline 5 & 2100 & 12.11 & 16.17 \\
\hline 5 & 4200 & 7.24 & 5.47 \\
\hline 5 & 6100 & 4.75 & 2.87 \\
\hline 7 & 2000 & 9.48 & 6.51 \\
\hline 7 & 4100 & 2.62 & 2.12 \\
\hline 7 & 5300 & 1.15 & 1.35 \\
\hline 8 & 1900 & 9.76 & 8.37 \\
\hline 8 & 3600 & 2.64 & 3.08 \\
\hline 8 & 5300 & 0.98 & 1.72 \\
\hline 9 & 2100 & 8.52 & 6.09 \\
\hline 9 & 4200 & 2.66 & 2.26 \\
\hline 9 & 6000 & 1.98 & 1.22 \\
\hline
\end{tabular}

numerical comparisons of the 3D-GILTT results against experimental data.

In Figure 2 the scatter diagram of model results against experimental data is presented and it can be observed that the present models in good agreement with experimental data.

In Table 7 some well-known statistical indices of models performances are reported. They are suggested and discussed in Chang and Hanna [17] and defined in the following way:

$$
\begin{aligned}
& \text { NMSE }=\overline{\left(C_{o}-C_{p}\right)^{2}} / \overline{C_{p}} \overline{C_{o}}, \\
& \mathrm{FA} 2=\text { data for which } 0.5 \leq\left(C_{p} / C_{o}\right) \leq 2, \\
& \mathrm{COR}=\overline{\left(C_{o}-\overline{C_{o}}\right)\left(C_{p}-\overline{C_{p}}\right) / \sigma_{o} \sigma_{p},} \\
& \mathrm{FB}=\overline{C_{o}}-\overline{C_{p}} / 0.5\left(\overline{C_{o}}+\overline{C_{p}}\right), \\
& \mathrm{FS}=\left(\sigma_{o}-\sigma_{p}\right) / 0.5\left(\sigma_{o}+\sigma_{p}\right),
\end{aligned}
$$

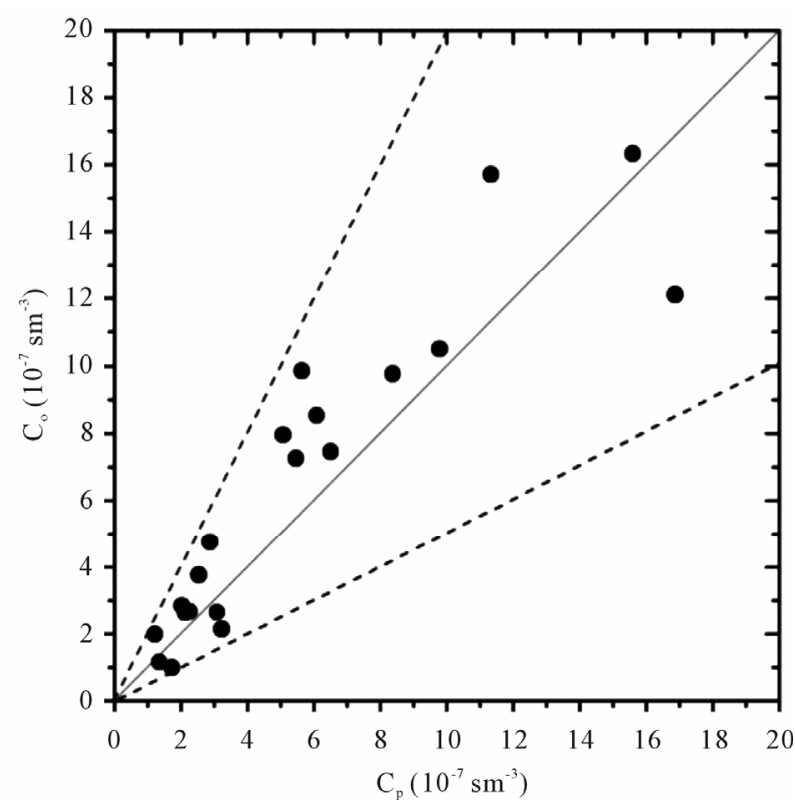

Figure 2. Observed $\left(C_{o}\right)$ and predicted $\left(C_{p}\right)$ scatter plot. Data between dotted lines correspond to $C_{o} / C_{p} \in[0.5,2]$.

Table 7. Statistical indices of model performances.

\begin{tabular}{cccccc}
\hline $\begin{array}{c}\text { Recursion } \\
\text { depth }\end{array}$ & NMSE & COR & FA2 & FB & FS \\
\hline 0 & 0.30 & 0.90 & 0.95 & 0.36 & 0.35 \\
1 & 0.12 & 0.91 & 1.00 & 0.11 & -0.03 \\
2 & 0.12 & 0.91 & 1.00 & 0.14 & 0.01 \\
3 & 0.12 & 0.91 & 1.00 & 0.14 & 0.02 \\
4 & 0.12 & 0.91 & 1.00 & 0.14 & 0.02 \\
5 & 0.12 & 0.91 & 1.00 & 0.15 & 0.03 \\
6 & 0.11 & 0.92 & 1.00 & 0.15 & 0.05 \\
\hline
\end{tabular}

where NMSE is the normalized mean square error, COR the correlation coefficient, FA2 is the fraction of data $(\%$, normalized to 1), FB the fractional bias, FS the fractional standard deviations. Subscripts o and $\mathrm{p}$ refer to observed and predicted quantities, respectively, $\sigma$ is the standard deviation, $C$ the concentration and the overbar indicates an averaged value. The statistical index FB says if the predicted quantities underestimate or overestimate the observed ones. FA2 is the fraction of $C_{o}$ values (normalized to 1) within a factor two of corresponding $C_{p}$ values. The statistical index NMSE represents the model values dispersion in respect to data dispersion. The best results are expected to have values near zero for the indices NMSE, FB and FS, and near one in the indices COR and FA2. So far, considering the statistical indices in Table 7 we can consider good the performance of the solution with the presented ABL parameterization. 


\section{Conclusions}

In recent years, it was presented in the literature, for the first time, a steady-state analytical solution for the advection-diffusion equation considering a vertically inhomogeneous PBL (with any restriction about the eddy diffusivity coefficients and wind speed profiles) based on the GILTT approach (Generalized Integral Laplace Transform Technique) $[2,3]$. Here we present a three-dimensional time-dependent solution with time-dependent vertical eddy diffusivity profiles that can be used in a time evolving turbulent boundary layer. Moreover, with the assumed ABL parameterization the model can be applied routinely using as input simple ground-level meteorological data acquired by an automatic network. Preliminary model performances evaluation confirms the reliability of the model results.

We also underline the hierarchical character of this methodology, in the sense that the solution of the threedimensional ADE problem is obtained from the solution of two-dimensional ones. Furthermore, this hierarchical character also prevails for problems with eddy diffusivity depending on time. In fact, for such problem the solution again attained from the solution of a set of problems with eddy diffusivity depending only on the vertical variable, having the main feature that the sources terms carry the time-dependency information of the eddy diffusivity. Therefore, from the previous discussion, we are confident that we have paved the road to construct a more realistic analytical solution for this kind of problem by now assuming a time dependency on the wind field too. We shall focus our future attention in this direction.

\section{Acknowledgements}

The authors thank CNPq (Conselho Nacional de Desenvolvimento Científico e Tecnológico) and FAPERGS (Fundação de Amparo à Pesquisa do Estado do Rio Grande do Sul) for the partial financial support of this work.

\section{REFERENCES}

[1] T. Tirabassi, "Operational Advanced Air Pollution Modelling," Pure and Applied Geophysics, Vol. 160, No. 1-2, 2003, pp. 5-16. doi:10.1007/s00024-003-8762-y

[2] D. Buske, M. T. Vilhena, B. Bodmann and T. Tirabassi, "Analytical Model for Air Pollution in the Atmospheric Boundary Layer," In: M. Khare, Ed., Air Pollution, Vol. 1, InTech, Rijeka, 2012, pp. 39-58.

[3] D. M. Moreira, M. T. Vilhena, D. Buske and T. Tirabassi, "The State-of-Art of the GILTT Method to Simulate Pollutant Dispersion in the Atmosphere," Atmospheric Research, Vol. 92, No. 1, 2009, pp. 1-17. doi:10.1016/j.atmosres.2008.07.004

[4] G. Adomian, "A New Approach to Nonlinear Partial Dif- ferential Equations," Journal of Mathematical Analysis and Applications, Vol. 102, No. 2, 1984, pp. 420-434. doi:10.1016/0022-247X(84)90182-3

[5] G. Adomian, "A Review of the Decomposition Method in Applied Mathematics," Journal of Mathematical Analysis and Applications, Vol. 135, No. 2, 1988, pp. 501-544. doi:10.1016/0022-247X(88)90170-9

[6] S. Wortmann, M. T. Vilhena, D. M. Moreira and D. Buske, "A New Analytical Approach to Simulate the Pollutant Dispersion in the PBL," Atmospheric Environment, Vol. 39, No. 12, 2005, pp. 2171-2178. doi:10.1016/j.atmosenv.2005.01.003

[7] D. M. Moreira, M. T. Vilhena, T. Tirabassi, D. Buske and R. Cotta, "Near Source Atmospheric Pollutant Dispersion Using the New GILTT Method," Atmospheric Environment, Vol. 39, No. 34, 2005, pp. 6290-6295. doi:10.1016/j.atmosenv.2005.07.008

[8] J. C. Kaimal, J. C. Wyngaard, et al., "Turbulence Structure in the Convective Boundary Layer," Journal of the Atmospheric Sciences, Vol. 33, No. 11, 1976, pp. 21522169.

doi:10.1175/1520-0469(1976)033<2152:TSITCB $>2.0 . C O$ ;2

[9] S. J. Caughey and S. G. Palmer, "Some Aspects of Turbulence Structure through the Depth of the Convective Boundary Layer," Quarterly Journal of the Royal Meteorological Society, Vol. 105, No. 446, 1979, pp. 811-827. doi:10.1002/qi.49710544606

[10] D. M. Moreira, M. T. Vilhena, D. Buske and T. Tirabassi, "The GILTT Solution of the Advection-Diffusion Equation for an Inhomogeneous and Nonstationary PBL," Atmospheric Environment, Vol. 40, No. 17, 2006, pp. 3186-3194. doi:10.1016/j.atmosenv.2006.01.035

[11] S. E. Gryning and E. Lyck, "Atmospheric Dispersion from Elevated Source in an Urban Area: Comparison between Tracer Experiments and Model Calculations," Journal of Climate and Applied Meteorology, Vol. 23, No. 4, 1984, pp. 651-654. doi:10.1175/1520-0450(1984)023<0651:ADFESI $>2.0 . C$ $\underline{\mathrm{O} ; 2}$

[12] S. E. Gryning, A. A. M. Holtslag, J. S. Irwin and B. Siversten, "Applied Dispersion Modelling Based on Meteorological Scaling Parameters," Atmospheric Environment, Vol. 21, No. 1, 1987, pp. 79-89. doi:10.1016/0004-6981(87)90273-3

[13] T. Tirabassi and U. Rizza, "Boundary Layer Parameterization for a Non-Gaussian Puff Model," Journal of Applied Meteorology, Vol. 36, No. 8, 1997, pp. 1031-1037. doi:10.1175/1520-0450(1997)036<1031:BLPFAN $>2.0$.C $\underline{\mathrm{O} ; 2}$

[14] G. A. Degrazia, H. F. Campos Velho and J. C. Carvalho, "Non-Local Exchange Coefficients for the Convective Boundary Layer Derived from Spectral Properties," Contributions to Atmospheric Physics, Vol. 70, No. 1, 1997, pp. 57-64.

[15] H. A. Panofsky and J. A. Dutton, "Atmospheric Turbulence," John Wiley \& Sons, New York, 1984.

[16] J. S. Irwin, "A Theoretical Variation of the Wind Profile 
Power-Low Exponent as a Function of Surface Roughness and Stability," Atmospheric Environment, Vol. 13, No. 1, 1979, pp. 191-194. doi:10.1016/0004-6981(79)90260-9
[17] J. C. Chang and S. R. Hanna, "Air Quality Model Performance Evaluation," Meteorology and Atmospheric Physics, Vol. 87, No. 1-3, 2004, pp. 167-196. doi:10.1007/s00703-003-0070-7 